\title{
Vitamin-B3-Überdosis?
}

\section{Akute Hepatitis durch zu viele Energydrinks}

Ein 50-jähriger Bauarbeiter hatte gerade noch einmal Glück: Vier bis fünf Energydrinks am Tag hatten seine Leber fast ruiniert. Ursache war vermutlich eine Niacin-Überdosis bei einer chronischen HCV-Infektion.

Die Annahme, Kräuter und Vitamine seien bedingungslos gesund, hält sich hartnäckig in der Bevölkerung und gibt immer wieder Anlass, den Schweizer Arzt und Mystiker Paracelsus mit seinem "alles ist Gift, allein die Dosis macht's“"zu zitieren. Seinen Paracelsus nicht gelesen hat offenbar auch ein 50-jähriger Bauarbeiter aus Florida. Mit vier bis fünf Energydrinks täglich wollte er seinen beschwerlichen Arbeitsalltag etwas aufpeppen. Letztlich hat er sich damit aber fast die Leber ruiniert.

\section{Ausgeprägte Gellbsucht}

Der Mann stellte sich in einer Klinik mit ausgeprägter Gelbsucht, Bauchschmerzen, Übelkeit und Erbrechen vor, berichten Ärzte um Dr. Jennifer Harb von der Universität in Gainesville. Zunächst habe er noch an eine Erkältung gedacht, als sich sein Haut gelb und sein Urin dunkel färbten, habe er jedoch eine ernsthafte Erkrankung erwogen und die Hilfe der Ärzte beansprucht.

Der Patient verneinte Leberprobleme in der Vergangenheit, auch seien solche in seiner Familie nicht aufgetreten. Er gab an, weder illegale Drogen noch Tabak oder übermäßig Alkohol $\mathrm{zu}$ konsumieren, auch habe er in letzter Zeit weder rezeptpflichtige noch rezeptfreie Medikamente eingenommen. An seiner Ernährung habe er ebenfalls nichts geändert - mit einer Ausnahme: Seit drei Wochen trinke er täglich vier bis fünf Energydrinks.



Die Ärzte bestätigten einen skleralen Ikterus und eine allgemeine Gelbsucht, zudem ertasteten sie einen harten rechten Oberbauch, fanden aber keinen Aszites, keine Asterixis oder andere Zeichen einer chronischen Lebererkrankung.

Laboruntersuchungen ergaben stark erhöhte Aminotransferase-Werte (AST: 1800 U/l, ALT: 1200 U/l), enorme Bilirubinkonzentrationen $(10 \mathrm{mg} / \mathrm{dl})$, aber auch deutlich zu hohe Werte für Vitamin B12 $(>2000 \mathrm{pg} / \mathrm{ml})$ und Folsäure $(>20 \mathrm{ng} / \mathrm{ml})$. Die Nierenfunktion war hingegen nicht eingeschränkt.

Im Ultraschall erwies sich die Leber als echogen, aber ohne Zirrhose, die Gallenblasenwand war verdickt, was die Ärzte auf die vorliegende akute Hepatitis zurückführten. Diese konnten sie mit einer Leberbiopsie bestätigen, dabei zeigten sich bereits Nekrosen und eine Cholestase.

\section{HCV plus Vitamin B3 als Ursache?}

Bei Antikörpertests fand das Team um Harb zwar Hinweise auf eine HCV-Infektion, die Antikörpertiter sprachen aber eher für eine chronische und nicht für eine akute Erkrankung. Andere typische Hepatitisursachen wie eine ischämisch bedingte Verletzung, eine Autoimmunerkrankung oder einen Morbus Wilson konnten die Ärzte ausschließen, auch für eine alkohol- oder medikamentenbedingte Toxizität fanden sie keine Hinweise, sodass sie den Grund im Überkonsum von

4-5 Dosen waren zuviel...
Energydrinks vermuteten. Für einen exzessiven Konsum sprachen neben den Patientenangaben auch die hohen VitaminB12- und Folsäurewerte. Oft enthalten Energydrinks hohe Konzentrationen solcher Substanzen.

Drei Tage nach dem Absetzen der Drinks gingen die Beschwerden zurück, die Transaminase- und Bilirubinwerte verdoppelten sich jedoch vom ersten bis zum vierten Tag nach der Klinikeinweisung und sanken dann ebenfalls. Auffällig war das disproportionale AST/ALTVerhältnis von etwa 2:1.

\section{Normale Kontrollwerte}

Aus Angst vor einem Leberversagen behielten die Ärzte den Patienten sechs Tage in der Klinik. Bei einer Kontrolle zwei Wochen später waren die Leberwerte wieder im Normbereich.

Energydrinks enthalten viel Koffein, Phenylalanin, Citicolin, Taurin, N-Acetyl-Tyrosin und B-Vitamine. Als hepatotoxisch ist davon nur Vitamin B3 (Niacin) bekannt. Allerdings werden Transaminaseerhöhungen erst bei Dosen jenseits der $500 \mathrm{mg} / \mathrm{d}$ erwartet, der Patient kam aber durch die Energydrinks allenfalls auf 160-200 mg/d. Möglicherweise waren hier seine chronische HCV-Infektion von Bedeutung oder aber Interaktionen mit weiteren Inhaltsstoffen der Drinks, die in der Regel diverse Kräuterauszüge enthalten.

Patienten mit bestehenden Leberschädigungen sollten daher lieber einen zurückhaltenden Konsum von niacinreichen Energydrinks pflegen, raten Harb und Mitarbeiter.

Generell empfehlen sie Ärzten, nach dem Konsum von Supplementen, kräuterhaltigen Produkten und Arzneien sowie Energydrinks zu fragen, wenn sich keine klaren Ursachen für eine akute Hepatitis aufspüren lassen.

(Thomas Müller)

Harb JN et al. Rare cause of acute hepatitis: a common energy drink. BMJ Case Rep, online 1. November 2016 